\title{
Cortical Responses to Local Electrical Stimulation of Retina
}

\author{
Robert W. Doty and Frances Ross Grimm ${ }^{1}$ \\ Department of Physiology, University of Michigan, Ann Arbor, Michigan
}

Received December 26, 1961

\begin{abstract}
Extensive cortical excitation from punctiform photic stimuli has been difficult to interpret because of intraoptic diffusion of light. Direct electrical stimulation of the exposed retina was thus used in this attempt to determine the degree of spatial elaboration of stimuli in the retinocortical system. Multichannel CRO recording from cortex and optic tract show two types of response in anesthetized or "midpontine" animals. The "early" response is identical in form to potentials elicited by optic tract stimuli, but has a latency of 2 to $5.3 \mathrm{msec}$, depending upon distance of intraretinal conduction. Constancy of timing in the cortical spike sequence despite varying arrival latencies shows that this well-known series of spikes is not generated as a consequence of conduction velocity differences in optic nerve fibers. Early potentials can be highly restricted in cortical location and, save for their predominance in the lateral dorsal region of marginal gyrus, generally conform in distribution to Talbot and Marshall's scheme of retinocortical topographical relations. "Late" responses are complex, but most are similar to photically elicited responses in form and latency. Their threshold is usually lower than that for early responses. Their cortical distribution for a given retinal locus greatly exceeds that of the early response, sometimes including ipsilateral cortex even to stimulation of nasal retina. Since optic tract potentials continue for over $100 \mathrm{msec}$ after the 1-msec retinal pulse, it is possible that some of the spatial elaboration of the late response occurs in the retina.
\end{abstract}

\section{Introduction}

On anatomical $(13,14)$ and physiological $(12,17)$ grounds it is accepted that the retina is represented on the cerebral cortex of the cat in a rather precișe topographical manner. However, punctiform photic

1 Supported by Research Grant B-1068 and Training Grant 2B-5252 from the National Institute of Neurological Diseases and Blindness, National Institutes of Health, United States Public Health Service. Dr. Grimm held a USPHS Postdoctoral Fellowship; her present address is: Hawthorn Center, Northville, Michigan. Dr. Doty's present address is: Center for Brain Research, University of Rochester, Rochester 20, New York. 
stimuli are able to elicit potentials in large areas of cortex (7). Interpretation of this unexpectedly diffuse responsiveness is difficult because of the possibility of light-scattering in the optic media. Considerable neural elaboration of a focal stimulus might occur and still be dismissed as a technical artifact.

The problem of light-scattering can be avoided by using local electrical excitation of the retina. The topographical arrangement of the retinocortical projection is confirmed by this procedure, but in addition responses are again found which greatly exceed expectations for a topographically organized system.

\section{Method}

Data were obtained from about fifty cats. Most of them were maintained under moderately deep Nembutal anesthesia, but the results were checked on animals prepared under ether with electrolytic decerebration at the midpontine level (1) and destruction of the trigeminal nerve.

On a few occasions concentric bipolar stimulating electrodes were introduced into the intact eye through a hypodermic needle penetrating the sclera. A series of experiments was also done in which the intact eye was exposed and freed of orbital tissue so that stimulating electrodes could be applied to any point on the sclera. Both of these methods of stimulation gave results similar to those to be reported for the open eye preparation, but were generally unsatisfactory; the former because of difficulty in controlling electrode placement, the latter because of the very high currents required to stimulate the retina through the sclera. The following preparation was thus used in the majority of experiments. The cornea was removed and the iris carefully cut with fine scissors at several places along its radii. Such radial cuts severed the constrictor iridae without undue hemorrhage. The anterior lens capsule and lens were then removed and the posterior lens capsule and vitreous humor repeatedly incised. The vitreous humor was gently removed by suction, exposing the interior of the eye to direct vision. Retinal detachment was not a problem, but sometimes an eye could not be used because it gradually collapsed. Secretions continued to accumulate in the globe and were removed by suction. To prevent excessive shortcircuiting of electrodes most work on the ventral retina was done in eyes sewn to a metal ring and rotated 45 to $90^{\circ}$ to bring the stimulated ventral quadrant free of any pool of fluid. Potentials evoked at the cerebral cortex by light flashes were monitored throughout each experiment. They showed 
no particular change upon opening the eye, and usually remained stable for several hours $\left(8 \frac{1}{2}\right.$ hours in one instance). The unused eye was kept covered.

The best retinal stimulating electrodes for the open eye were made from $0.2 \mathrm{~mm}$-diameter (32-gauge), insulated nichrome wires, each bent into a tight loop from the face of which the insulation was removed. Interelectrode distance was kept at about $1 \mathrm{~mm}$. Grass S-4 stimulators provided 1-msec pulses to the electrodes or, for photic stimulation, drove a miniature tungsten filament lamp (General Electric No. 327; Ref. 7) to produce $200-\mathrm{msec}$ flashes. Repetition rate for photic or electrical stimulation was 0.3 per sec. Position of the retinal electrodes was recorded in millimeters from the optic disc and in relation to the dorsal retinal artery and ventral border of the tapetum. Stimulation was done routinely with the animal partially dark-adapted.

The cortex was widely exposed and kept under oil. High viscosity silicones were also tried and provided satisfactory protection of the cortex without the necessity of making a skin pouch to retain them, but repositioning of electrodes was made difficult by this material. Potentials were recorded from the cortical surface through $0.5-\mathrm{mm}$ diameter silver ball electrodes, positioned under rack and pinion control in the Johnson stereotaxic machine, and referred to a screw in the lambdoidal ridge. Position of cortical electrodes is designated in terms of the coordinates given in the atlas of Jasper and Ajmone-Marsan (10). The letters " $\mathrm{A}$ " and "P" indicate millimeters anterior to the interaural plane. In five animals the medial surface of the hemisphere was explored following acute or chronic removal of medial tissue from the other hemisphere. Records from the optic tract were obtained from paired $0.3-\mathrm{mm}$ diameter wires with uninsulated tips separated by about $1 \mathrm{~mm}$. The electrodes were often placed by hand and left unsupported in the brain, but such procedure seemed to result in more rapid deterioration of the potentials than when the electrodes were placed and held with a carrier. In most experiments Tektronix type-122 preamplifiers, with time constants set for linearity of response between $2 \mathrm{kc}$ and $0.8 \mathrm{per} \mathrm{sec}$, were used with a 5-channel oscilloscope.

\section{Results}

A 1 -msec electrical pulse ( 1 to 15 volts) applied to a small area of the retina elicits two types of response at the cortex. One we have termed the "early" response. It has a wave form identical with that 
seen for stimulation of the optic nerve, and arrives within 2 to $5.3 \mathrm{msec}$, depending upon location of the stimulus. The others, herein called the "late" responses, are more variable. They frequently are similar to the photically elicited response in form, latency and amplitude at a particular cortical location, but may also be much smaller, or appear as a complex series of waves, or range in time of onset from 10 to over $100 \mathrm{msec}$.

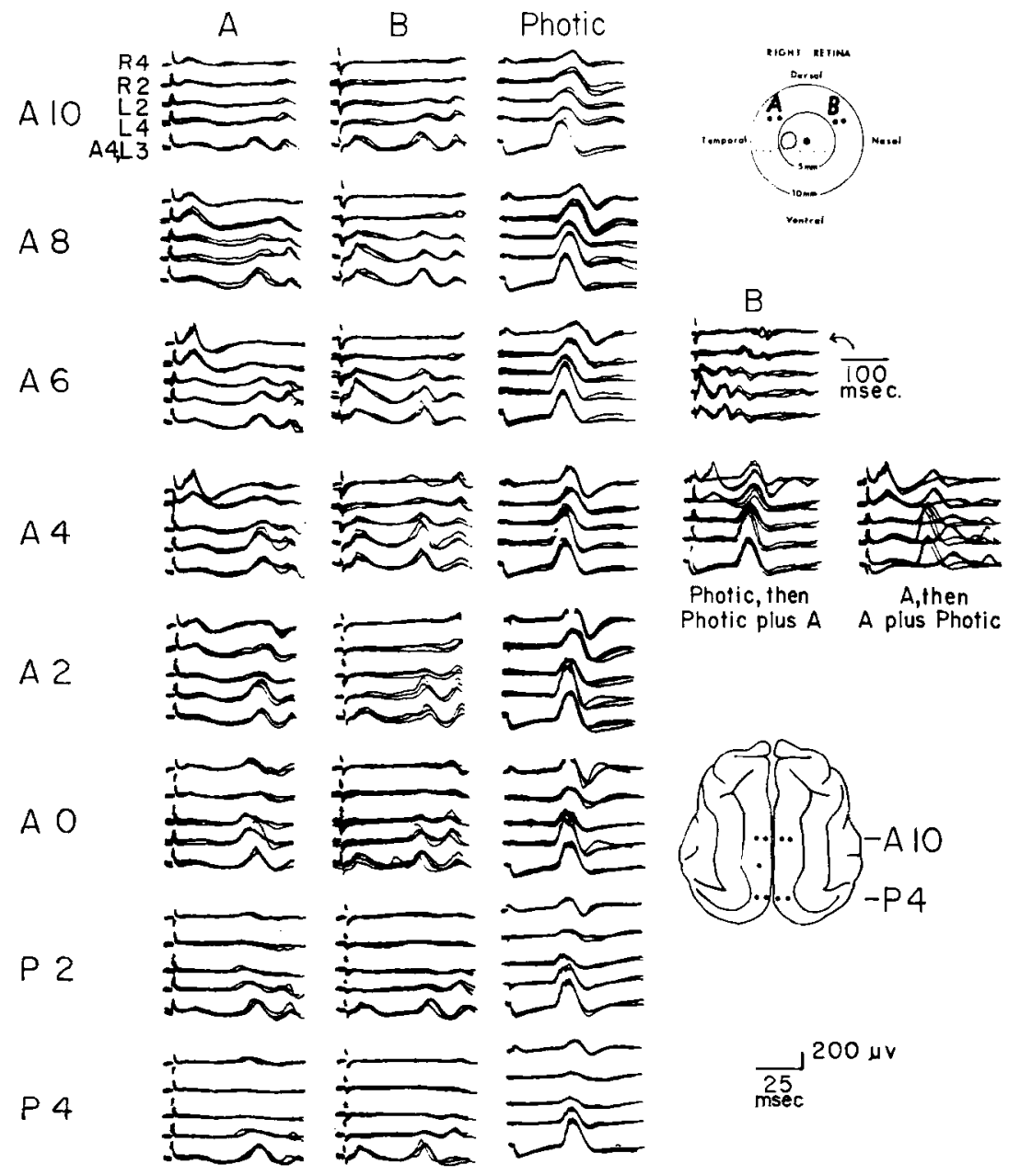

Fic, 1. 


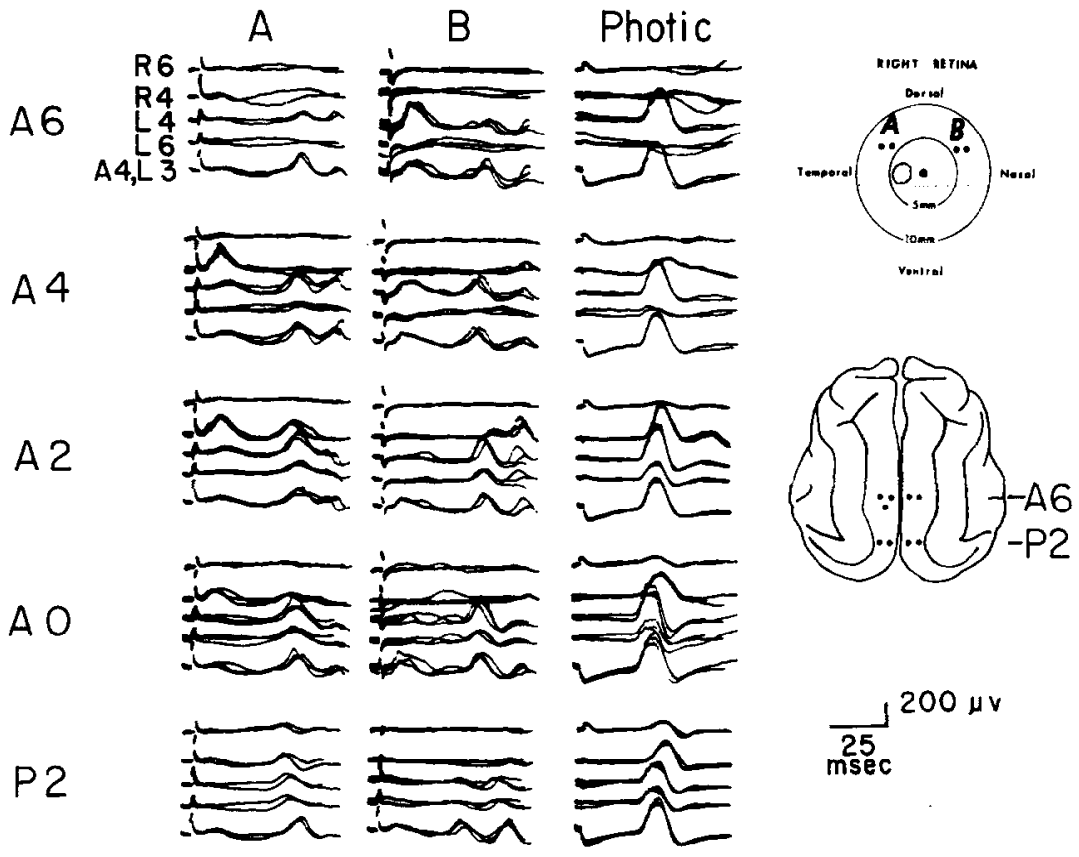

FIG. 2.

FIGs. 1 and 2. Cat 537 under Nembutal, with trigeminal nerve cut. Concurrent comparison of responses at various cortical loci to diffuse photic stimulation and electrical stimulation of retinal sites marked A and B, stimulus 6 volts, 1 msec. Series begun 2 hours after opening eye; 1.5 hours to complete. Temperature at end of run: $34^{\circ} \mathrm{C}$. Lowest (fifth) trace in each group taken from electrode fixed at A-4, L-3, serving as control for moment to moment variability. Note the early response to stimulation with retinal electrodes "A" appears only on anterior ipsilateral cortex whereas that to " $\mathrm{B}$ " is in contralateral cortex and slightly more restricted. In each instance a series of late waves appears over wide areas of ipsi and contralateral cortex, tending to be most pronounced (for " $A$ " especially) where the early response is lacking. In Fig. 1 slower sweep at far right shows responses occurring more than $100 \mathrm{msec}$ after stimulation at "B". At A-4 a photically elicited potential abolishes late response to electrical stimulation at " $\mathrm{A}$ " and early electrical response depresses that to photic stimulation. In Fig. 2 note absence of response laterally in anterior cortex, and appearance of late response to " $B$ " at A-0 to A-4 in the ipsilateral hemisphere. Latency of early response (on faster sweeps than shown) was $4 \mathrm{msec}$ for "A", $5 \mathrm{msec}$ for "B"; about $36 \mathrm{msec}$ for the first late response and $28 \mathrm{msec}$ for photic response. Positivity of active electrode signalled by upward deflection in all figures. 

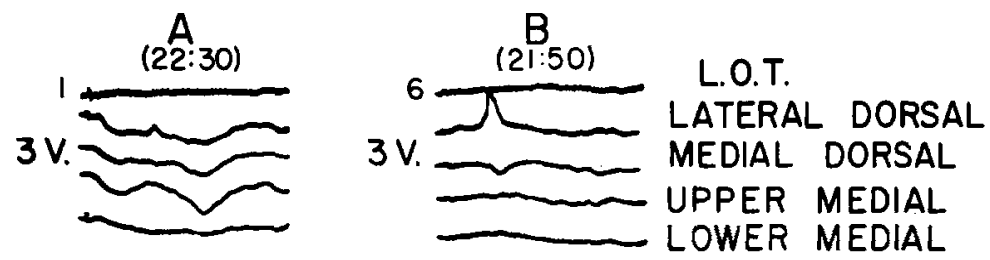

A 8
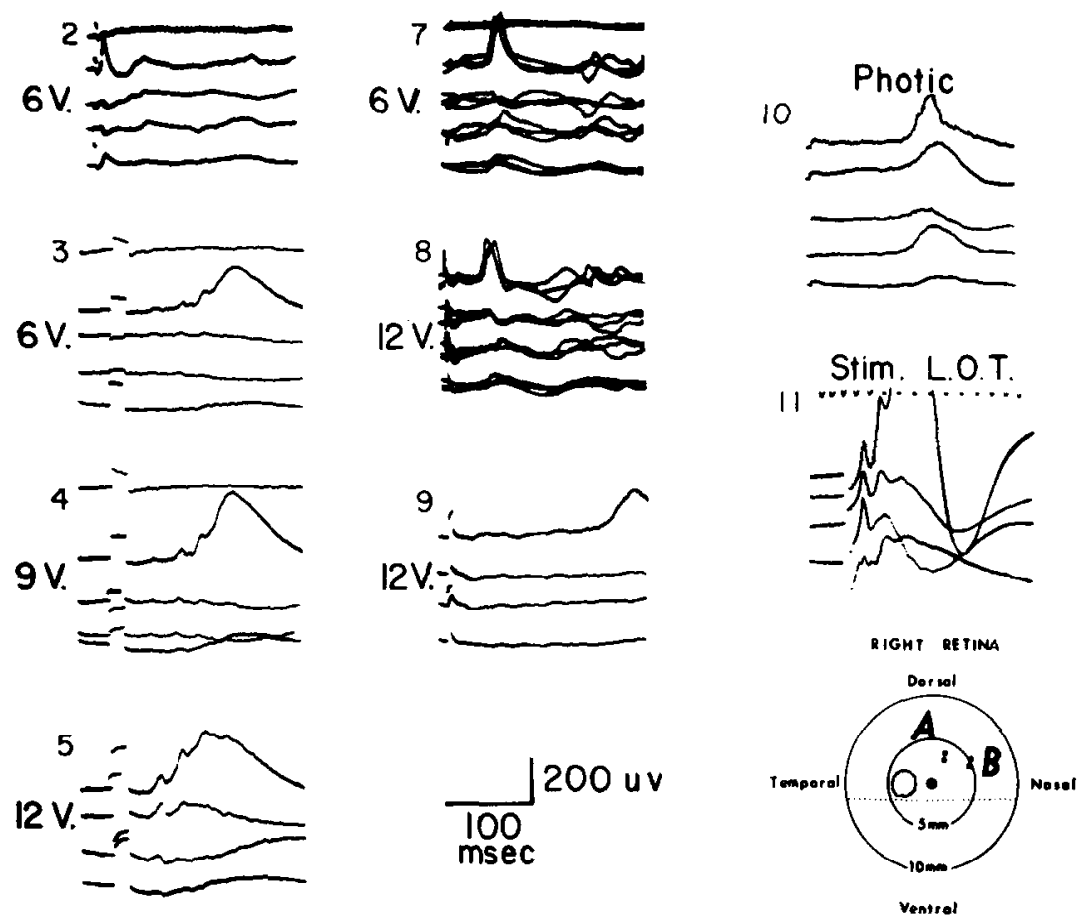

FIG. 3. Cat 863, under Nembutal. Early and late responses from retinal points A and $B$ stimulated within a few minutes of each other. Right visual area removed 1 week earlier, providing access to medial marginal gyrus ("upper medial" trace) and splenial gyrus ("lower medial" trace) at A-8 level. Optic tract trace (L.O.T.), at half gain of others, showed no evidence of early or late potentials at higher gain or stimulus voltage although responding to photic stimulation (record "10"). Time for records " 3 ," " 4 ," "5" given by 1000 per sec signal on record " 11 "; records " 9 " and "10" at sweep speed $6 \times$ faster than $100-\mathrm{msec}$ calibration of other records. Note shift in latency of early response as stimulus intensity increases (records " 3 ," " 4 ," "5"), but maintenance of spike interval equal to that for L.O.T. stimulations ("11"). Also note difference in form of early potentials on the medial surface. 
Characteristics and Cortical Distribution of Early Responses. When the stimulating electrodes are applied to the optic disc or, with stronger stimuli, within 2 to $3 \mathrm{~mm}$ of the disc (Fig. 3), the response at cortex begins within $2.0 \mathrm{msec}$ (usually less). Early responses of longer latency

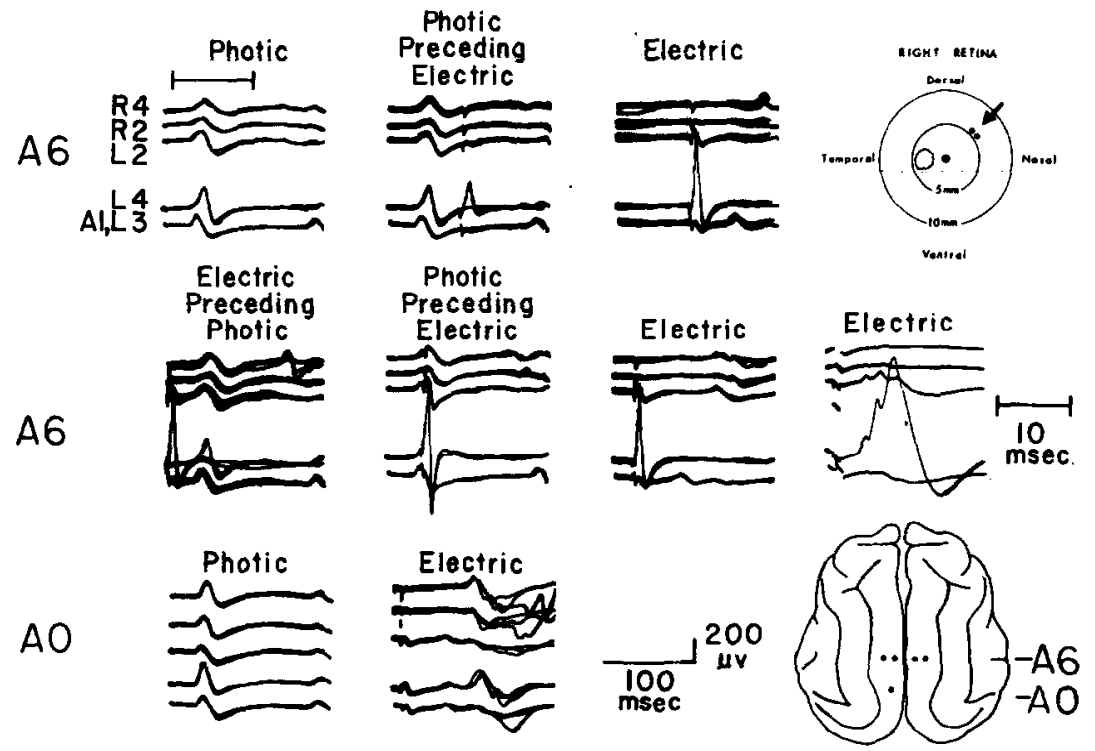

FIG. 4. Cat 490, under Nembutal. Interactions of responses to photic and electric stimuli. Sharply localized, early response at A-6, L-4 is greatly depressed by response to antecedent flash-onset and vice versa. When responses to electric and photic stimuli are phased to occur together, there is slight summation, particularly of the negative phase. First late responses to electrical stimulation are depressed by antecedent photic respenses; some very late responses may be enhanced as at R-4, left of middle row. Fast sweep shows early response has form typical of that for optic nerve stimulation. Latency is $3.8 \mathrm{msec}$, threshold 1 volt for 0.5 -msec pulse; 15 volts, 1 -msec pulse used here. At this stimulus intensity amplitude of early potential was down $50 \%$ at $\mathrm{A}-4$ and $\mathrm{A}-8,85 \%$ at $\mathrm{A}-10, \mathrm{~L}-2$, and absent elsewhere. Posteriorly (A-0) early response is replaced by late responses, and some appear in the ipsilateral hemisphere with latencies as long as $70 \mathrm{msec}$.

are inferred to arise from stimulation of distal fibers (Figs. 1, 2, 3, 4, 6). The longest latencies observed were $5.3 \mathrm{msec}$ from electrodes about 8 to $10 \mathrm{~mm}$ from the disc. Allowing for some effective spread of the stimulus around the electrodes, this addition of $3.3-\mathrm{msec}$ latency is in excellent agreement with that expected from Dodt's data (6) for the conduction velocities of the faster intraretinal fibers. A rough estimate 
of $5 \mathrm{~m}$ per sec for intraretinal conduction velocity is also provided by recording in the same animal the latency differences at cortex for responses elicited from stimuli at various distances along a retinal radius. The times between spikes $2,3,4$, or 5 [in the terminology of Chang and

A 5
6 Volts

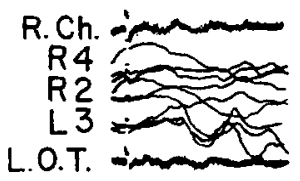

9 Volts

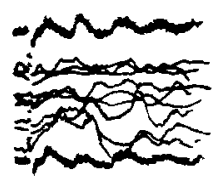

A 5
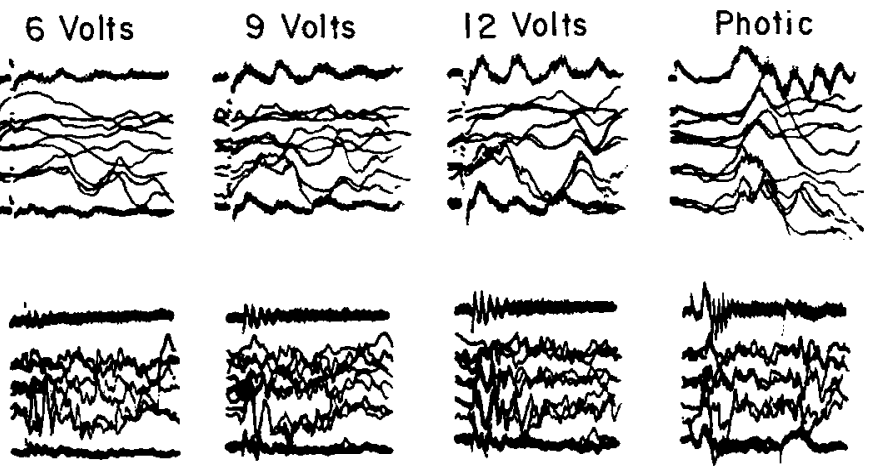

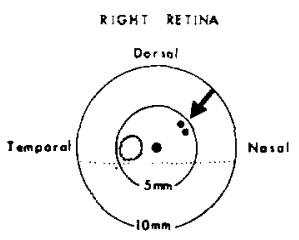

Ventrol
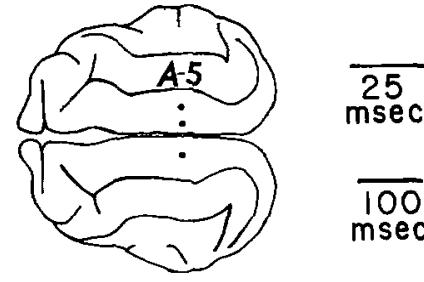

$200 \mu v$

FIG. 5. Cat 546 with midpontine transection. Series of responses elicited in optic tract and cortex of unanesthetized preparation by diffuse photic and punctate electrical stimulation of retina, immediately after opening eye. Upper row, faster sweep. The damped oscillations in chiasm and tract to single, 1-msec electrical pulses are about $13 \mathrm{msec}$ peak to peak whereas those to photic stimulation are about $7 \mathrm{msec}$. On original records increase in background activity for over $100 \mathrm{msec}$ following electrical stimulus can be seen and is apparent here as a thickening of the base line at slower sweeps for right chiasm (R. Ch.) at 9 and 12 volts and with photic stimulation. High gain for optic tract and chiasm. Artifacts indicating "on" and "off" of illumination can be seen in chiasm and tract traces in lower right record. Rectal temperature $39^{\circ} \mathrm{C}$.

Kaada (4)] of the cortical potential remain constant irrespective of initial latency (Fig. 3).

As shown in Figs. 1, 2, and 4, the early cortical response may be highly restricted in location. Naturally the extent of cortex involved increases as the electrodes approach the disc and subtend a greater number of fibers, or when the stimulus intensity is increased to excite 
a larger retinal area. In some instances it is possible to obtain relatively well-localized cortical potentials even when the retinal stimulating electrodes are less than $2 \mathrm{~mm}$ from the disc, but of course no statement can then be made concerning the retinal origin of the fibers involved.

When the electrodes are placed $3 \mathrm{~mm}$ or farther from the disc, the cortical region in which the early response is found bears a rough topographical relation to the retinal region stimulated. In so far as they
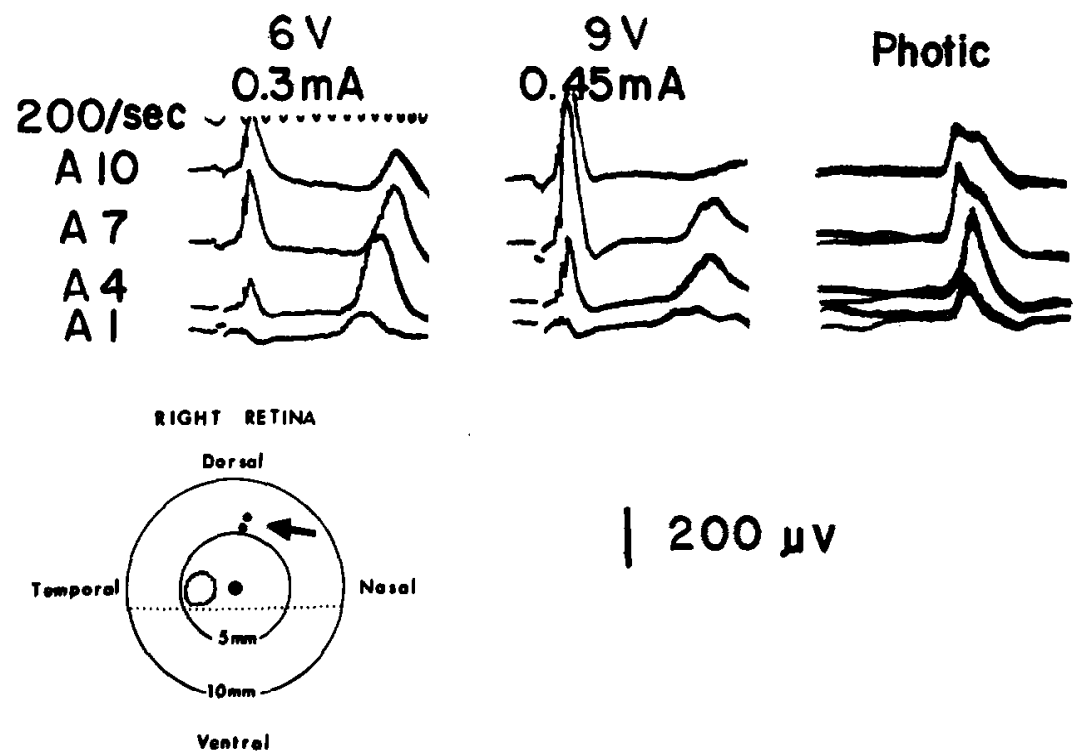

\section{| $200 \mu \mathrm{V}$}

Fic. 6. Cat 868, under Nembutal. Reciprocity of early and late responses, and progressive increase in latency of late response with distance anterior along midmarginal gyrus. Nonlinear sweep displays some detail of early response on same trace with late response.

can be demonstrated in this manner, the retinocortical relations observed are in agreement with those found by Talbot and Marshall $(12,17)$. There was some overlap of cortical potentials evoked by stimulation of adjacent retinal sectors (e.g., Fig. 1 in Ref. 8), but this would be difficult to quantify. The location of the largest cortical response moves posteriorly from the stereotaxic plane $\mathrm{A}-12$ to about $\mathrm{A}-0$ as the stimulus is brought from the most dorsal portion of the retina down to the ventral boundary of the tapetum.

This orderliness in projection of dorsal-ventral location for the dorsal 
retina upon the dorsal surface of marginal gyrus could be readily demonstrated. A comparable nasal-temporal progression could not be found except for the gross division of nasal and temporal retina projecting to ipsi and contralateral hemispheres. Failure to detect nasaltemporal ordering as easily as that for dorsal-ventral might be the result of the fact that the early responses appear predominantly in the lateral-lying, "high-amplitude" strip of marginal gyrus ("region A" in Ref. 7). Of early, well-localized potentials, only three were found outside this region (in cats 582,647, and 881). The response in cat 647 had a latency of $5.3 \mathrm{msec}$ and was $14 \mathrm{~mm}$ from the midline on the ventral tip of postlateral gyrus at P-6, contralateral to stimulation of a region $3 \mathrm{~mm}$ ventral to the tapetum and $6 \mathrm{~mm}$ nasal of the disc. In cat 881 the response was found on the medial surface at the ventral border of the splenial gyrus at the A-0 to A-2 level with a latency of $5.2 \mathrm{msec}$ upon stimulation $5 \mathrm{~mm}$ temporal of the disc and $3 \mathrm{~mm}$ into the tapetum, i.e., just lateral to area centralis, in the ipsilateral eye. The stimulated region in cat 582 was similar to that in cat 881 but probably slightly dorsal and medial to it since responses occurred in both hemispheres. A series of simultaneous 4-channel records from the marginal gyrus showed the ipsilateral response to be sharply localized on the marginal gyrus only $2 \mathrm{~mm}$ lateral to the midline whereas the contralateral response was in the usual location $4 \mathrm{~mm}$ from the midline. Both were at the A-4 level.

Characteristics of Late Responses. The late response cannot be obtained from stimulation of the optic disc or nerve. In the retina proper the threshold for the late response typically is less than that for the early and averages about 3 volts for a 1 -msec pulse. On the three occasions when the current was measured at threshold it was less than $0.1 \mathrm{ma}$. The late response requires a longer pulse duration than does the early; e.g., changing the stimulus from $1.0 \mathrm{msec}, 6$ volts to $0.1 \mathrm{msec}$, 9 volts elicited the same early response but the late potential, present with the longer pulse, was absent with the shorter.

With increasing stimulus intensity ( 3 to 6 volts) the late response increases in amplitude and becomes more regular, the latency usually diminishing slightly. With further increase ( 6 to 9 or 12 volts) the late response often decreases and may even disappear (Fig. 6). If the positions of the recording and stimulating electrodes are appropriate, such decrease in the late response with increasing stimulus is accompanied by the appearance and growth of an early response (Fig. 6). This is a frequent occurrence since in about seventy per cent of the preparations both 
early and late responses could be found concurrently at one or another cortical locus. In only three of fifty-seven instances did an increase in stimulus intensity beyond that necessary for one late response produce a new, later sequence of responses. Double waves are sometimes seen, however, with latencies of about 20 and $40 \mathrm{msec}$, respectively, and the earlier of the two may have a threshold somewhat higher than the second. Double and multiple responses also occur sometimes when longer d-c pulses are used (Fig. 7). Late responses of widely different latencies can appear concurrently in the two hemispheres (Fig, 1).

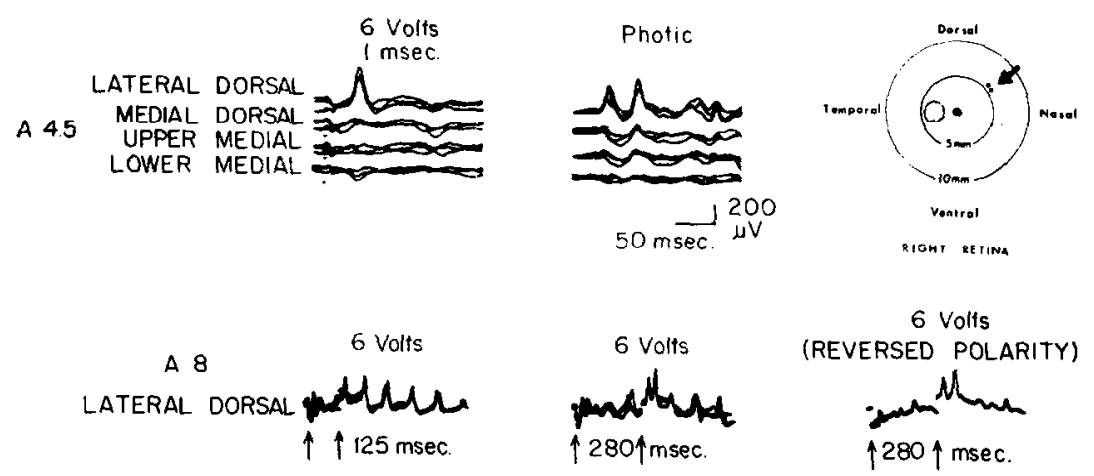

Frg. 7. Cat 881 , under Nembutal. Series of late responses elicited by d-c pulses of varying duration. Note double form is also characteristic of photic response in this animal. Medial portion of right hemisphere removed acutely.

From the foregoing and from the figures it can be seen that the form and latency of the late response is extremely variable. In the great majority of cases, however, the late response is similar in form to the photic response, although it is seldom as large, and has a latency roughly $10 \mathrm{msec}$ longer, i.e, about 30 to $40 \mathrm{msec}$ to onset of the late response. When early and late responses are obtainable concurrently, the late response always has a much wider distribution than does the early response. On the other hand, the late response is always more restricted in its distribution than is the response to a flash of diffuse light.

Like the early responses, the late activity occurs most consistently in the region A-0 to A-8, 4 to $5 \mathrm{~mm}$ lateral to the midline; but it usually exceeds this region and often appears bilaterally. It is, however, restricted to marginal, splenial, and postlateral gyri. Stimulation of the nasal retina can elicit late responses in the ipsilateral hemisphere (e.g., Figs. $1,2,4)$, with latencies as short as $33 \mathrm{msec}$ in some cases. So too can 
stimulation in the temporal retina, eliciting a well-localized ipsilateral early response, at the same time elicit widespread contralateral late responses of 30- to 40-msec latency. Stimulation in the ventral retina often evokes late responses in the anterior marginal gyrus at the A-6 to A-8 plane.

In certain cases, e.g., Fig. 6, the late response has a progressively increasing latency from one cortical region to another. The latency difference is equivalent to an intracortical conduction velocity of $1 \mathrm{~m}$ per sec. Such systematic latency differences, however, are exceptional.

Interactions of Early, Late, and Photic Responses. Both early and late responses are reduced or abolished if they are preceded by a response to light (Figs. 1, 4). The late response is the most susceptible to such suppression and can sometimes be held in abeyance by continuous light shone into the eye. A 3-msec flash (which is very feeble because of the heating time of the tungsten filament) can abolish a late response for several hundred milliseconds. Suppression of the late response at the cortex is accompanied by similar absence of the late response in the optic tract.

As noted above, the early and late responses tend to be mutually exclusive and an increase in the former usually diminishes the latter. A strong early response also forestalls the appearance of a photic response in cortex occupied by the early response (e.g., Fig. 4). A late response preceding a photic or early response can diminish them, but this effect is minimal compared to that in the reverse direction.

Both the early and late responses have very long recovery periods so that a second response to a repeated stimulus is entirely missing for 90 to $200 \mathrm{msec}$. Recovery may not be complete even after $700 \mathrm{msec}$.

Nature of Late Responses. Artifactual effects somewhat resembling the late response can be produced in a "monopolar" recording situation by facial reflexes resulting from stimulation of ophthalmic trigeminal fibers. This is particularly troublesome when stimulation is applied through the sclera. The spurious nature of such potentials is readily detected because of their nonattenuating distribution over the cortex. Nevertheless it was necessary to show that the late response was exclusively related to the optic system. This was done by recording cortical late responses after surgical section of the trigeminal nerve (Figs. 1,2) or after section of the brain stem anterior to the trigeminal entrance (Fig. 5 and Ref. 8). Local destruction of the retina subtending electrodes producing early or late responses abolishes these responses, 
and no late response results from stimulation of the optic disc. Retinal injury is not a necessary factor for producing late responses since it is possible to elicit them in typical form by stimulation through the sclera of the intact eye.

A retinal origin for a major portion of the cortical late response is suggested by the fact that corresponding potentials can be recorded from the optic tract. In several cases a distinct potential could be observed in the tract 1.5 to $4 \mathrm{msec}$ prior to a late response at the ipsilateral cortex. This latency difference is the same as that recorded between tract and cortex for a photically elicited response. The responses are usually more complex, however, and the absolute correspondence between tract and cortical potentials is then uncertain, as in Fig. 5. Lack of response in recordings from the optic tract cannot be taken as evidence for a central origin of the late response since tract recordings sometimes fail to show the early response traversing the tract (Fig. 3). Additional evidence that significant excitation can traverse the optic tract without gross electrical sign is found with experimental occlusion of the retinal circulation: the recorded tract response fails about $20 \mathrm{sec}$ prior to loss of the photic response at the superior colliculus. ${ }^{2}$

As mentioned above, the late response can often be dissociated from the early by its lower threshold. This nevertheless leaves some possibility that an undetected early response might be present. Appearance of a late response in a situation where occurrence of the early is extremely unlikely was observed in one experiment. The late response, 3 hours after opening the eye and placing the electrodes, had increased in latency from 45 to $55 \mathrm{msec}$ and become unstable. An early response remained unchanged at a point $2.5 \mathrm{~mm}$ lateral to the cortical point from which the late response was recorded. With dual pulse stimulation at a pulse interval of $100 \mathrm{msec}$ the early response was absent to the second pulse, but concurrently the late response, when failing to appear to the first pulse, was often fully developed to the second.

"Multiple" responses $(7,9,16)$ to a flash of light usually invert from negativity to positivity a few tenths of a millimeter above the point within the cortex at which inversion occurs for the "primary" response. When multiple responses to photic stimuli and late responses to electrical stimuli are present at the same cortical point, an electrode withdrawn through the cortex shows the inversion of the late response occurs with that of the primary photic response rather than the multiple response.

2 R. W. Doty and D. S. Kimura (unpublished). 


\section{Discussion}

Early Response. There is no reason to doubt that the early response arises from excitation of ganglion cells or their fibers in the immediate vicinity of the retinal electrodes. The extremely restricted cortical distribution of some of the early potentials and their general location confirms the basic idea and arrangement of a retinocortical topographical projection $(12,13,14,17)$. However, the predominance of these potentials in the laterally situated, "high-amplitude" region of marginal gyrus still leaves some serious difficulties in the interpretation of this topography.

The fact that the time relations between the series of spikes recorded at cortex does not vary with variations in overall latency of the group (Fig. 3) strongly supports the idea that these spikes represent a process inherent in the cortex $(2,11)$. The evidence exemplified in Fig 3 is incompatible with Chang's proposal (3) that the cortical spike series arises from differences in conduction velocity of optic nerve fiber systems projecting to cortex. Dodt's figures (6) show intraretinal conduction velocity differences for group I and group II fibers such that the slower fibers should lag the faster at the optic disc by $2.5 \mathrm{msec}$ if both were stimulated $5 \mathrm{~mm}$ distal to it. No cortical potential was found which could be attributed to this slower fiber group.

Late Response. The form and latency of most of the late responses could be explained if it were simply assumed that the electrical pulse activated photoreceptors. Indeed, it would be difficult to account for the long latency of the potentials seen traversing the optic tract were this not the case. Even so, no explanation can be offered for some of the later tract potentials, nor for the occasional potentials of 10 -msec latency which may be too fast for the usual photic pathway.

If the electrical pulse in its effect actually does imitate a photic stimulus, then some later cortical potentials similar to photically elicited "multiple" responses $(7,9,16)$ would be expected. Perhaps some of the electrically elicited potentials with latencies greater than $60 \mathrm{msec}$ should be explained in this way; but this is a gratuitous assumption and definitely is not indicated for the late responses of 20 - to $50-\mathrm{msec}$ latency. That the latter are not basically attributable to intracentral elaboration of an early response is evidenced: (a) by the absence of late responses to optic disc stimulation; (b) by the general reciprocal relation between early and late responses; (c) by the dissociation of the 
two types of responses with threshold stimuli or during refractoriness of the early response; and (d) by similarity of the intracortical potentials for photic and late responses as distinct from the multiple response.

The magnitude, duration and complexity of the optic tract potentials following a 1-msec electrical pulse to the retina (e.g., Fig. 5) suggest a diffuse intraretinal elaboration of the excitation. Direct evidence for an intricate intraretinal network is seen in the work of Crapper and Noell (5) who observed repeated bursts in retinal ganglion cells of rabbits following brief electrical stimuli. Their results indicate also that it might be possible for a series of potentials to originate from the excited zone. Potentials seen in the optic tract in the present experiments thus need not necessarily represent a spatial elaboration; but, of course, neither is this possibility eliminated. Conducted "slow" potentials have been observed $2 \mathrm{~mm}$ away from a highly localized electrical stimulus in carp retinae (15).

On the other hand, photically evoked potentials can spread for many millimeters into partially isolated cortex $(7,8)$ at the same velocity as that indicated for the late response in Fig. 6. Furthermore, destruction of a retinal sector, from which electrical stimulation produced localized early responses at a particular cortical locus, can be accomplished with very little change in the photically elicited response from that cortical locus. Thus central elaboration is also possible. Preliminary results, however, indicate that a cut of several millimeters can be made in the retina between stimulating electrodes and optic disc without loss of the late response. This suggests both a retinal and central extension of the excitation.

\section{References}

1. Batini, C., G. Moruzzi, M. Palestini, G. F. Rossi, and A. Zanchetti, Effects of complete pontine transections on the sleep-wakefulness rhythm: the midpontine pretrigeminal preparation. Arch. ital. biol. 97: 1-12, 1959.

2. Bishop, G. H., and M. H. Clare, Sites of origin of electric potentials in striate cortex. J. Neurophysiol. 15: 201-220, 1952.

3. ChANG, H-T., Functional organization of central visual pathways. Research Publ. Assoc, Research Nervous Mental Disease 30: 420-453, 1950.

4. Chang, H-T., and B. KaADA, An analysis of primary response of visual cortex to optic nerve stimulation in cats. J. Neurophysiol. 13: 305-318, 1950.

5. Crapper, D. R., and W. K. NoELx, Retinal responses to direct electrical stimulation. Physiologist 3 (3): 42, 1960.

6. DoDt, E., Geschwindigkeit der Nervenleitung innerbalb der Netzhaut. Experientia 12: $34,1956$. 
7. Doty, R. W., Potentials evoked in cat cerebral cortex by diffuse and by punctiform photic stimuli. $J$. Neurophysiol. 21: 437-464, 1958.

8. Doty, R. W., Functional significance of the topographical aspects of the retinocortical projection. In "Neurophysiologie und Psychophysik des visuellen Systems," Jung, R. and Kornhuber, H. (eds.), pp. 228 247; Heidelberg, Springer-Verlag, 1961.

9. Fleming, T. C., and E. V. Evarts, Multiple response to photic stimulation in cats. Am. J. Phy:iol. 197: 1233-1236, 1959.

10. Jasper, H. H., and C. A JMONE-Marsan, "A Stereotaxic Atlas of the Diencephalon of the Cat," 15 pp., 54 pl; Ottawa, National Research Council of Canada, 1954.

11. Landau, W. M., and M. H. Clare, A note on the characteristic response pattern in primary sensory projection cortex of the cat following a synchronous afferent volley. Electroencephalog. and Clin. Neurophysiol. 8: 457-464, 1956.

12. Marshall, W. H., and S. A. Talbot, Recent evidence for neural mechanisms in vision leading to a general theory of sensory acuity. Biol. Symposia (Visual Mechanisms) 7: 115-164, 1942.

13. Mnnkowski, M., Über die Sehrinde (Area striata) und ihre Beziehungen zu den primären optischen Zentren. Monalschr. Psychiat. Neurol. 35: 420-439, 1914.

14. Minkowski, M., UUber den Verlauf, die Endigung und die Zentrale Repräsentation von gekreuzten und ungekreuzten Sehnervfasern bei einigen Säugetieren und beim Menschen. Schweiz. Arch. Neurol, Phychiat, 6-7: 201-303, 1920.

15. Motokawa, K., E. Yamashita, and T. Ogawa, Interaction of slow potentials of the retina evoked by photic and electric stimuli. Tôhoku J. Expil. Med. 71: 67-77, 1959.

16. Talbot, S. A., and W. H. Marshall, Multiple responses in the optic tract and optic cortex of cat. Am. J. Physiol. 129: 478, 1940.

17. Talbot, S. A., and W. H. Marshalt, Physiological studies on neural mechanisms of visual localization and discrimination. Amer. J. Ophthal. 24: 1255-1264, 1941. 